\title{
2.3 Zum Konzept einer Pathologie als Physiologie der Krankheit. Pathogenie und Ätiologie bei Karl Wilhelm Stark (1787-1845)
}

\section{A. Bauer}

\subsubsection{Einführung: Zur Situation der Medizin im Biedermeier}

Das 19. Jahrhundert stellt in der Geschichte des ärztlichen Denkens, Wissens und Handelns eine deutliche Zäsur dar, die unsere heutige Medizin von den älteren Konzepten der Heilkunde trennt. In den Jahren um 1850 hat sich ein säkularer Paradigmawechsel von der empirisch oder spekulativ orientierten Heilkunde zur Medizin als angewandter Naturwissenschaft vollzogen, den man durchaus revolutionär nennen kann. Schrittmacher für diesen Wandel waren die "Geburt der Klinik"1 am Ende des 18. Jahrhunderts einerseits, die Entwicklung der Grundlagenfächer Physiologie und Pathologie auf naturwissenschaftlicher Basis andererseits.

Der folgende Beitrag soll sich mit einem Pathologen und Kliniker beschäftigen, der zur Zeit jenes Umbruchs gelebt hat, nämlich dem Jenaer Professor der Medizin KARL Wilhelm STARK (1787-1845) aus dem Umfeld der „Naturhistorischen Schule". Diese in Deutschland zwischen etwa 1825 und 1845 maßgebliche Schule gruppierte sich um den aus Bamberg stammenden Kliniker JoHANN LUKAS SCHÖNLEIN (1793-1864), der von 1840-1859 Leiter der Berliner Charité und Leibarzt des preußischen Königs FRIEDRICH WILHELM IV. war. Die Naturhistorische Schule im Sinne ScHÖnLEINs arbeitete klinisch und streng empirisch, unter Verzicht auf eine a priori akzeptierte allgemeine Krankheitslehre. Durch exakte Beobachtung einzelner Krankheitsfälle wollte man stattdessen zur Konstruktion abstrakter Krankheitsbilder, zur Bildung von Krankheitsarten und Krankheitsfamilien, endlich zu einem ,natürlichen System" der Krankheiten fortschreiten. Da sich die „Naturhistoriker" der Abstraktheit ihrer Krankheitseinheiten bewußt waren, entwickelten sie Regeln für ihre Verfahrensweise. Diese Regeln wurden den Vorstellungen der naturphilosophischen Medizin aus der Zeit des Deutschen Idealismus um 1800 entnommen $^{2}$, als deren Grundannahmen zu nennen sind:

- Krankheiten sind Prozesse.

- Alle Krankheiten sind örtlich.

„Für die Abstraktion von idealen Krankheitsbildern bedeuteten diese Prámissen, daß zeitlich aufeinander folgende Phänomene zusammenhängend berücksichtigt werden mußten, und daß die Bewertung der Symptome an der Dignität der Organe und Gewebe orientiert wurde, auf die sie sich beziehen lieBen“*3. 
Neben der Aufstellung von Krankheitsbildern befabte sich die Naturhistorische Schule auch mit epidemiologischen Problemen sowie mit medizinhistorischen Untersachungen zur Geschichte einzelner Krankheiten (Historigche Pathologie)". Thre fahrenden Reprasentanten lassen sich weder als „Naturphilosophen" noch als "Naturwissenschaftler" in heutigen Sinn klassifizieren, vielmehr handeft es sich um eine eigenständige Richtuag rwischen diesen beiden Epochen, die zwar Grundgedanken der Naturphilosophie integrier, andererseits aber auch Vorarbeiten far die naturwissenschaftlich-experimentelle Forschung in der Pathologie geleistet hat. Sie erscheint als ein wesentlicher Teil dessen, was RoTHschuH mit den Schagwort „Deuische Biedermeiermedizin ${ }^{\text {th }}$ gekennzeichnet hat.

Im politischen Bereich wird unter dem Biedermeier die Zeit zwischen 1815 und 1848 verstanden, die Jahre der Realution und Restauration nach den Befreiungskricgen auf der eincn, des nach Demokratisierung strebenden „Vormilrz" auf der anderen Seite'. Beide Strömungen verdeutlichen die dialektische Widersprochlichkeit der Epoche, ein Phlinomen, das auch in kulturellen und in wissenschaftichen Bereich registriert werden kann. Ein innerer Rackrug in die Privatsphäre, die bürgerliche Idylle, verbunden mit wachsender Skepisis gegen abergreifende, mit Monopolanspruch auftretende Theorien oder Systeme bestimmi den Altag des forschenden Mediziners jener drei Jahrzehnte. „Man ist ... beseelt von Eifer, neue Erfahrungen zu machen. Man ist geradexu erfahrungssuchtig, es wird gesammelt, beschrieben, benannt, verglichen, klassifnziert. Mar halt sich in der Generalisierung betont zuruck, das Einzelne verdient eher das liebevolle Interesse als das Allgemeine. Man scheut die Spekula. tion und liebt die Beobachtung, man behatrt auf dem Vordergrindigen. Anatomie, Physiologie und Klinik bevorzugen die deskriptive Methode der Vergleichung, der erfahrungsgemliBen Korrelation der Erscheiaungen und Umstande. Man sucht nach empirischen Gesetzen, die moglichst mumerisch gesichert sein sollen.... Das Zeitalter ist .... noch fast, worexperimentell' zu nennen .... Dureh die Arztewelt zieht der Geist auberster Ernochterung, man ist theoriefeindlich und von grofier therapeutischer Skepsis erfullir"t.

\subsubsection{Kurzporträt von KAin. Wruncu StakK (1787-1845)}

Kahl. Witunl. StakK wurde am I8, 5, 178 ? in Jena geboren. Sein Vater JoHaNN Chkistian Srakk (1753-1811) war Ordinarius der Medizin an der Universitat Jena. Er diente der Herzogin ANNA AMALLIA (1739-1807) van SachsenWeimar sowie dem Dichter FrIEDRICH SCHILER (1759-1805) als Leibara: auch Goirthit wurde von ihm in medizinischen Fragen beraten. Kans. WiLHELM, der entgeborene Soln, studierte von 1804 bis 1807 an der Universitat Jena Medizin, vor allem unter der wissenschaflichen Obhut seines Valers. Nach dem Rigorosum ernannte ihn Herzog Kasa. Aucust (1757-1828) von Sachsen-Weimar zum Hofmedicus. Im Jahre 1811 warde Srark unter seinem

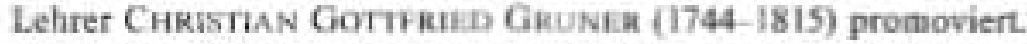

Von 1811 bis 1813 trat er eine in seiner Zeit abliche Weiterbildungsreise quer durch Europa an; Wien, Venedig, Bologna, Florenz, Rom, Paris und Ber- 
lin warea Zwischenstationen dieser Studien. Nach seinet Ruckkehr in die Heimat emannte ihn der Herzog zum Extraordinarius und Leibarzt. Als Leibara der Grobherrogin weilte SrAkK I814/15 in Wien und konnte so die politischen Umwalizungen, die auf dem Wiener Kongre 8 vorbereitet wurden, aus nächster Nahe verfolgen. Von 1815 bis 20 seinem Tod im Jahre 1845 wirkte STARK un. unterbrochen in Jens, hier vollzog sich sein allmahlicher, aber netiger beruficher Aufstieg: Zunllchst hielt er an der Universitat Vorlesungen tber Enzyklopaldie der Medizin, Algemeine Pathologiv und Therapie, Ophthalmologie, Allgemeine Chirurgie und Gerichtsmedizin, 1817 erfolgte die Ernennung zum Hofrat, 1826 wurde er Ordinarius, 1836 Geheimer Hofrat. Schlie日lich abernahm er 1838 nach dem Tod seines Vetters Johann Chisman STakk (17691837) die Mitdirektion der medizinisch-chirurgischen Landesheilanstalten, der Klinik, der Irrenanstait und des Entbindungshauses zu Jena. In dieser Eigen. schuft publizierte er 1839 einen „Plan zur innern Einrichtung und Verwaltung einer offentlichen Krankenanstalt", den wir an anderer Stelle untersucht ha* ben?. Am 15. 5. 1845 starb KaRL. WhLHEL STARK in Jena, drei Tage vö sei. nem 58. Gicburtstag.

Die Einordnung von SrakK unter die Reprilsentanten der Naturtistorischen Schule war lange Zeit umstritten; wahrend altere Medizinhistoriker ihn als den eigentlichen Begrander dieser Richtung betrachteten". lassen neuere Forschungsergebnisse erkennen, daB STARK weder personell noch inhaltlich in den engeren Kreis um ScuONLEIN gehörte". Es erscheint daher am sinnvollsten, ihn als einen Mediziner im Umfeld der Naturhistorischen Schule zu bezeichnen, der durch eine besondere Theorie bekannt wurde: den Versuch einer ontologischen Pathologie, in welcher er die Krankheit - nach der Art eines Analogiemodells - mit einem parasitischen Wesen verglich. Der erkrankte Organismus erscheint in diesem Modell als Träger und Nahrboden der Krankheit. Wir wollen im folgenden Stakks Theorie verfolgen, soweit sie fur uner Thema Pathogenie und Ätiologic relevante Kontequenzen birgt.

\subsubsection{Priazipien der ontologischea Pathologie}

Als Srapks Hauptwerke sind die "Pathologischen Fragmente" (zwei Bande 1824/25) sowie seine „Allgemeine Pathologie oder allgemeine Naturlehre der Krankheir" (1838, Neuauflage in zwei Bănden 1844/45) anzusehen. Wir wollen an dieser Stelle hauptsachlich auf die .Allgemeine Pathologie" in der Endfas. sung von $1844 / 45$ zuruckyreifen, da in diesen beiden Banden auf insgesamt 1600 Seiten die Theorie seiacr ontologischen Krankheitslehre systematisch und umfassend abgehandelt wird. Zugleich macht dieses Spatwerk Uatenchiede wie Ähnlichkeiten zur naturwissenschaftlichen Medizin der zweiten Jahrhunderthaifte deutlich, deren souveranster Vorkaimpfer, Rubour Vinchow (18211902), bereits 1847 auf der 25. Versammlung deutscher Naturforscher und Arzte in Aachen seinen ersten Vortrag .ober Entzîndune mit parenchymatösem Exsudate" hilt ${ }^{10}$.

In der 1838 entstandenen Vorrede verweist SтакK auf die besonderen Vor- 
teile seiner ,naturhistorischen Ansicht der Krankheir", indem er als deren hervorstechendstes Merkmal die Moglichkeit eines Anschlusses der Pathologie an die Physiologie beschreibt: „Die naturhistorische Ansicht der Krankheit nothigt ... geradezu, sich einer physiologischen Grundlage zu bedienen .... Nur mit tiefer BetrubniB wird der Patholog die grolle Strecke gewahr, welche die Physiologie bei ihrem gewaltigen Aufschwung ihrer Zwillingsschwester, der Pathologie, vorangeeilt ist, und die betrachtlichen Lacken, welehe die mangeinde Berâcksichtigung der Fortschritte jener in dieser gelassen hat .... Mein Bestreben ging daher ... dahin, die Pathologie auf Physiologie .... zu gründen, die letztere bei dem großen Vorsprung, welchen sie der erstern abgenommen, wo möglich einzuholen, und die specielien Lehren jener mit den entsprechenden physiologischen wieder ins Gleichgewicht 20 bringen ${ }^{+11}$.

Dieses Leitmotiv einer physiologischen Basis der Pathologie bekraftigt der Autor nochmals in der Vorrede zur zweiten Auflage 1844; sein Ziel bleibe es, „der Krankheit die Rechte und Gesetze des Lebens uberhaupt nicht blo 8 zo vindiciren, sondern sie an sich betrachtet als einen selbst voin normalen Leben weder dem Wesen, noch der Form nach, nur hinsichtlich ihrer individuellen Beziehung verschiedenen Vorgang darzustellen und somit .... ihre bloße Relativitat zur allgemeinen Anerkennung zu bringen, der Pathologie dadurch eine ... physiologische Bedeutung .... zu ertheilen und sie daher auch an den Fortschritten, welche Physiologie und ihre Hülfswissenschaften in der neuern Zeit machten, grobern Theil nehmen zu lassen ${ }^{i s}$.

\subsubsection{Krankheitsbegriff}

Die Entwicklung der Pathologie nach den Gesetzen der Physiologie - das ist die Zentralidee STARK5, die er im „ersten Fundamentalsatz der allgemeinen Pathologie" sogleich umsetzt:

..Krankheit ist Leben. Ohne Leben keine Krankheit. Sie erscheint daher.... nur als ein Autribut, als ein Zustand, ein Vorgang des Lebens ${ }^{+1}$. Damir werden bereits zwei komplementáre Aspekte in der Betrachtung pathologischer Phãnomene erfabt: Krankheit ist dem Leben zugeordnet 1, als Zustand (statischer Aspekt, ,pathos') sowie 2. als Vorgang (dynamischer Aspekt, nosos'). Die Krankheit stelit sich als nahere Bestimmung des konkreten Lebens dar, sie unterliegt den allgemeinen Naturgesetzen und ist aicht heteronom w denken.

Wie sieht STARK das Verhalitnis von pathos' und nosos' zur aegritudo', zum Kranksein? „Erkrankung. Krankseyn ist ... ein, von Krankheit selbst sorg* faltig $z$ unterscheidender Lebenczustand, welcher in dem gleichzeitigen Vorkommen .... verschiedener Lebensformen in einem .... Organismus und daher in der Vereinigung desselben zu einem scheinbaren Ganzen besteht .... Das Krankseyn ist daher ein gemischter ... Zustand eines organischen Wesens" ${ }^{-14}$. Und weiter: „Die Vereinigung ungleichartiger Lebensprocesse findet sich bei den aormalen Parasiten und ihrem Mutterorganismus" is.

Jetat ist das Schlagwort gefallen, mit dem STARks Pathologie immer wieder identifizien worden ist: „Parasitismus" als Extremform einer ontologischen Krankheitslehre, als spekulative Verirrung eines naturphilosophischen Epigo- 
nen. Es muB - zur Vermeidung von Fehlurteilen - aber stets das Motiv im Auge behalten werden, das ihn zur Formulierung seiner Theorie veranlaBt hat, nlimlich die Bearbeitung der Pathologie mit dem methodischen Arsenal det Physiologie ais einer „Physiologischen Pathologie* im wortlichen Sinn, denn .Krankheit (ist) ihrem Wesen nach auch Leben (und) ... demanch auch denselben Gesetzen ... unterworfen" is.

In Analogie zu lebenden Wesen schreibr STARK der Krankheit Selbsterhaltungs- und Regenerationsvermogen sowie Individualitat zu, ein Gedanke, den er bercits 1875 in einem Aufsatz uber .Individualitat des Krankheitspronesses" ausgeführt hat. Bereits in jener Abhandlung taucht die Idee der „Krankheitselemente ${ }^{4}$ auf, die einen Krankheitsprozel konstituieren. „Jeder .... KrankheitsprozeB besteht aus einer ... bestimmten Anzahl von einander verschiedener .... zur Einheit verbundener .... Krankheitselemente .... Der Verein der unmittelburen AeuBerungen der Krankheit (pathognomonische, wesentliche Symptome), ist die Form der Krankheit. Auf der Eigenthitmlichkeit dieser Combinationen pathognomonischer Symptome beruht der wesentliche Unterschied der einreinen Krankheitsformen" if

Als das Kardinalproblem der ontologischen Pathologie erweist sich die Frage nach dem Substrat der Krankheit. Srask postuliert, Krankheit sei wie jedes Leben - Materie und Kraft zugleich. Dabei konne das Hauptgewicht im Eineelfall mehr auf der stofflichen oder mehr auf der dynamischen Seire fiegen (Extremfalle wairen organische Millbildungen einerseits, Geistes: krankheiten ohne morphologisch fabbare Verlinderungen andererseits). Went Krankheit ein dem normalen Leben gleichender ProzeB ist, so besitzt sie ebenso wie jenes einen Anfang, eine gesetzmilBige Dauer und ein Ende, d. $h$. sie „stirbt .... eines natorlichen oder eines gewaltsamen Todes ${ }^{+1 \text {. }}$. Der cinzige Unterschied der Krankheit zu anderen Lebensprozessen besteht demnach darin, daB sie . immer ein anderes ... Leben ... voraussetzt, un, in und mit dem (sie) lebt. Sie ist also ein Parasirt ${ }^{40}$.

An diesem Punkt stellt sich die Frage, ob Stakks Krankheitsbegriff als Metapher, als Modell oder als „naturwissenschaftlich" zu wertende Realitat aufgefabt werden muB. Der Autor entscheidet dieses wissenschaftstheoretisch bedeutsame Problem nicht vollig eindeutiz. Denn unmiltelbar nach der eben zitierten scheinbar apodiktischen Feststellung alubert er sich zum parasitischen Charakter, der keineswegs der Krankheit allein zukomme, bei dem es sich vielmehr um ein universelles Phänomen handele: „Streng genommen ist der Parasitismus eine jedem Leben zukommende Eigenschaft ... Selbst die hơhem Thiere und der Mensch sind Parasiten des Erdorganismus ... Aber der Parasitismus selbst ist wieder ein sehr relativer Zustand ${ }^{4020}$.

Aus diesen Sazzen Ieiten wir folgende Deutung ab: Der Begrift "Parasit" darf nicht biologisch verstanden werden, abo nicht als Klassifizierung der Krankheit im Rahmen einer botanischen oder zoologischen Kategorie des Linnéschen Systems. Mit dem mparasitischen Charskter" hebt STAgK lediglich cine seiner Auffassung nach jeder lebendigen Strukrur inhlirente Eigenschaft hervor, die bei der Krankheit in besonderem Malle ausgeprägt zu sein scheint. Aus einer solchen Betrachtungsweise resultieren rwei Vorteile, námlich erstens die Möglichkeit ciner physiologischen Bearbeitung der Pathologie und zwei. 
tens cin leicht operationalisierbarer Zugang zum Phänomen Krankheit. Man kann STARKS Interpretation der Krankheit daher als Analogiemodell bezeichnen, bei dem - pars pro toto - eine wesentliche Eigenschaft modellhaft vereinfachend fur die ganze Erscheinung gesetzt wird ${ }^{21}$. Dall er sich dabei eines Analogiemodells bedient, kann bei einem naturphilosophisch geschuiten Geist nicht erstaunen; ebenso wie die Ant cines Modells mul aber auch stets dessen Motivation beachtet werden, ohne die es unverstandlich bleibt. Das Ziel von Stanks Modell ist die Anbindung der Pathologie an die Theorie der Physiologie einerseits, Fortschritt für die arztliche Diagnostik und Therapie andererseits $^{22}$.

Eine grundlegende Konsequenz des „Parasitismus" besteht im Postulat einer Lokalpathologie sensu stricto. Dies ist unmittelbare Folge der Individualitat sowie des parasitischen Charakters der Krankheit. Dadurch findet auch die spexifische Relation zwischen befallenem Organ und der Krankheit ihre plausible Erklarung: „Wie .... jedes Organ nur gewissen Parasiten zum Aufenthalisorte dient, so giebt es auch nur den Sitz für gewisse Krankheiten abt+23. Eine Allgemeinkrankheit sei mit dem Leben unvereinbar, da der Parasit dann dic Existenz seines Trägers zerstören wïrde. Krankheit könne auch keine Reaktion des Korpers gegen ein exogenes Agens darstellen, weil es sich bei einer solchen Reaktion um etwas ZweckmaiBiges, bei der Krankheit aber um etwas Unzweckmalliges handele.

Wesentlich fü die Stellung der Disziplin Pathologie im diagnostisch-therapeutischen Prozeb ist die Sicherung ihres Gegenstandsbezirkes. Dazu gehònt eine Kiarung den Verhalitnisses von ,pathos', , nosos' und, aegritudo:. Nach STAkK fallen der Pathologie zwei Aufgaben zu: In erster Linie mul sie den vom erkrankten Menschen 20 abstrahierenden KrankheitsprozeB beschreiben und klassifizieren, in zweiter Linie hat sie die morphologischen bzw. funktionellen Verlanderungen und Störungen des betroffenen Organismus zu untersuchen. Die Pathologie STAkxs versteht sich demnach primär als eine „NaturIehre der Krankheit", wie es der volle Wortlaut ithres Titels ankandigt. DaB es sich hierbei nicht um eine „Krankheitsbiologie* handelt, folgerten wir bereits aus der Struktur des gewahlten Analogiemodells.

\subsubsection{Pathogenie und Ätiologie}

„In sofern der Vorgang der Krankheitsentstehung selbst von den ihn bedingenden Ursachen unterschieden werden kann, .... labt sich auch die Lehre von der Entstehung der Krankheit (Pathogenie im engern Sinne) von der Lehre der Krankheitsbedingungen oder urstichlichen Momente (Aetiologie) trennen. Wenn jene die allgemeinsten Bedingungen, die Art und Weise und das Wesen des Entstehens der Krankheit zu entwickeln hat, so muB diese die ursilchlichen Momente, welche den hinlanglichen Grund des Entstehens der Krankheit bilden, angeben. Doch befalit man auch beide Lehren unter der gemeinschaftichen Benennung der letziem (Aetiologie) $)^{-12}$,

So definiert Stank die beiden Begriffe Pathogenie und Atiologie. Wahrend er jedoch dem Gebiet der Atiologie 545 Seiten widmet, begnìgt er sich bei der 
Pathogenie mit 29 Seiten; das entspricht einem quantitativen Verhaltrais von 19:11

\section{Puthogente}

Die Möglichkeit der Kraskheitsentstehung beruht auf einer doppeilen Bedingung, nlmalich AuBlenwelt und Orgunismus. Die Wirkung der Aubenwelt wird als âuliere Schldlichkeit (potentia nocens), die pathogene Pradisposition des Organismus als Krankheitsanlage (causa interna) bezeichnet: potentis nocens und causa interne mllssen als ursachliche Momente zusammenwirken, um den hinreichenden Grund für die Krankheitsentstehurg bilden zu kônnen, „Der individuelle Organismus sorgt als solcher stets far seine Selbaterhaltung .... Das aubere unachliche Moment giebt also die date Gelegenheit zur Erkrankung. Daher nennt man auch die lubere Bedingung der Krankheit vorzugsweise Gielegenheitsursactie derselben (causa occasionalis) ${ }^{\text {ala }}$,

Das homöostatische Gleichgewicht rwischen Individunu und Umwelt wird also zunachst durch eine Veranderumg der letrieren gestort. Der Organismus setrt sich gegen eine solche Turbulenr regulativ mur Wehr, wobei drei unter. schiedliche Ergebnisse moglich sind: Entweder gelingt tie Kompensation der exogenen Nose vollatandig oder nur teilweise oder gar aicht. Im dritten Full kann im Organismus bei vorhandener Anlage eine Krankheit entstehen; Schldtichkeit und Anlage mossen dabei aber zuelnander Beziehung haben. also eine qualitative Sperifitat aufweisen. . Wirkt sin FiafluB auf eine ihm nicht entsprechende Anlage. so vermag er sie aicht zar wirklichen Krankheit auszubilden ... Ist er ilir der Qualist nach entgegengesetat, so hebt er sie sogar selbst auf .... Je stârker das eine undchliche Moment thiltig ist, in desto geríngerm Grade braucht es das andere zu seyn, um die Krankheit wirklich zu er. zeugen $^{* 2 n}$, Cansa ocessionalis und causa interna verhalten sich demnach komplementair wie Schlosseł und Schlod zaeinander; reine „Uawelticider" oder ausschließliche „Erbkrankheiten" sind fur STAkx folglich undenkbar.

Fine pathogenetische fiedeutung komma issbesondere den Krankheitsatrien zu. den Eintrittspforten for exogene Noxen. Dazu zahlen vor allea die au. Beren und inaeren Korperoberflachen (Haut, Speiselanal, Respirationsorgane. Harn- und Geschlechtsorgane), aber auch das Nervensystem, welches weniger für materielle als fur dynamische Schadlichkeiten empianglich ist. Das Krankheitsatrium fungiert als Verminter der auBeren Noxe, sofern es selbst eine entsprechende Anlage besitzt. Ohne diese Anlage wird die Schadlichkeit durch das Atrium abgewehrt und gelangt nicht zur Entfaltung-

Den Vorgang der eigentlichen Pathogenese vergleicht STARK im Rahmen seines Analogiemodells mit einer Zeugung, wobei die Krankheitsanlage das motterlich-weibliche, die auBere Schadlichkeit das valterlich-männliche Prinzip vertritt. Die sich nun entwickelnde Krankheit eleicht daher einem kleinen Embryo, der sich von seiner Zeugung an autonom weiterentwickelt. Das befallene Individum hat lediglich die Funktion eines Nahrbodens für den jungen Krankheitsparasiten zu ubernehmen, seine Reaktionen gehôren aber nicht zum Krankheitsprozeb selbst. 


\section{Ätiologie}

Die ursächlichen Momente für die Krankheitszeugung (Patho-genese im wōrtlichen Sinn) bestehen, wie bereits angefuhrt, aus dem weiblichen Prinzip der Krankheitsanlage (causa interna) und dem mannlichen Prinzip der bußeren Schädichkeit (causa occasionalis), Die Krankheilsanlagen gliedern sich nun wie folgt:

Normale Krankheitsanlagen

Genterische Krankheirsanlage

- Racenanlagen

- Nationelle Krankheitsanlage

- Familienanlage

- Zeitliche Krankheitsanlage der Gattung

- Räumliche Krankheitsaniage der Gattung

Individuelle Krankheitsanlage'

- Constitution

- Symmetrische Anlage

- Temperament

- Geschlechtsanlage

- Entwickelungsveränderungen

Specielle Krankheitsanlage

Abnorme Krankheitsanlage

Abnorme Anlage der Krankheit

Abnorme Anlage des Kranken

- Abnorme generische Anlage

- Abnorme individuelle Anlage

- Abnorme specielle Anlage

Die ,normalen' (besser: primären) Krankheitsanlagen definiert STARK so:

"Die generische Krankheirsanlage hat das Individuum mit andern seiner Gattung gemein. Sie wird daher von den àitern Pathologen gemeinschaftliche natürliche Krankheitsanlage' (seminia morborum naturalia communia, GAUB) genannt ${ }^{+27}$.

Jedes Individuum ist eigen geartet und unterscheidet sich durch seine Eigenthümlichkeiten von andern Individuen seines Gleichen ... Wir haben diese (mit RerL) die individuelle Krankheitsanlage genannt ${ }^{* 23}$.

„Jedes Organ ist ... ein in gewisser Hinsicht verschiedenes und bis auf einen gewissen Punct unabhägiges Leben .... und so gleichsam wieder .... ein individueller Organismus im Individuum .... Als eine solche relative Totalitit ... hat sic ihre eigene Krankheitsanlage ${ }^{+2 \%}$.

Auf diese Weise enthält die Untergliederung der Erbweit - hierarchisch abgestuft - je ein phylogenetisches, ein ontogenetisches und ein organogenetisches Prinzip. Dabei beinhalten die Obberlegungen zur Organpathologie durchaus schon histopathologische Tendenzen. So nennt STARK beispielsweise als Voraussetzung für einen Katarrh das Vorhandensein einer Schleimhaut, für das Auftreten von Krâmpfen die Existenz von Muskelgewebe. 
Die zweite Gruppe der Krankheitsanlagen bilden die ,abnoraen' (besser: sekundaren); diese setzen einen anderen prỉexistenten Krankheitsprozeß voraus, der seinęneits zu neuer Erkrankung disponiert. Modern formuliert entsprliche eine solche Anschauung der Konstruktion pathogenetischer Ketten beziehungsweise der klinischen Erscheinung ,Muttimorbiditar*. Auch hier kann nach generischer, individueller und spezieller Anlage differenziert werden, sowohl auf seiten der Krankheit wie des Kranken.

Den Krankheitsanlagen werden nun die Gelegenheitsursachen (causae occasionales) als Umwelteinflasse im weitesten Sinn ecgenubergestellt. Waren jedoch die Krankheitsanlagen auf knapp 60 Seiten abzuhandeln, so benotigt Srank fur die Darstellung der auBeren Schldilichkeiten 460 Seiten, also den naheru achtfachen Ruum. Die Umwelteinflasse lassen sich in vier Hauptgruppen trennen:

\section{Dynamische Schädlichkeitua}

- physisch-dynamische

- organisch-dynamische

- psychisch-dynamische

Chemische Schdillichieiten

- absolute Iublers

- relative aubere

Mechaninche Schaidlichkeiten

- absolute AuBere

- relative äubere

Complicirte, gemischte Schädlichheiven

Unser Schema ist naturlich extrem vereinfacht und gibt den taxonomischen Feinbav des sehr differenzierten Systems nur unvollkommen wieder. So watren die physisch-dynamischen Schadlichkeiten wu untergliedern in Einflisse der Gravitation, der Gestirne, des Lichts, von Farben, Temperatur, Tages- und Jahreszeiten, Klima, Atmosphäre und Witienang, von Gerüchen, Schall und Erdmagnetismus; die gesamte physikalische Umwelt stellt cin pathogenes Potentiul dar. Dengegenuber treten die biologischen Wechselwirkungen organischer Körper aufeinander (organisch-dyaamische Potenzen) quantitativ eher in den Hintergrund, ebenso die Störungen des Gefahls, des Willens und des Erkenntnisvermogens (psychisch-dynamische Schlidlichkeiten).

Den ober ihre unsichtbare Kraft wirkenden dynamischen Gefahrenquellen schlieBen sich die chemischen Noxen an, die in Gasen, Nahrungsmittein, Getranken, Tabak, Medikamenten und Giften (cxogen) sowie Sekretions- und Exkretionsprodukten (endogen) bestehen können. Die dritte Kategorie der mechanischen Schadlichkeiten urnfaft vor allem Bekleidung- und Bewegungsprobleme, wahtrend die vierte das soziale Umfeld des Erkrankten (Wohnverbaltnisse und Beruf) behandelt ${ }^{2}$,

Die Atiologie der Krankheit beruht also in STARKs ontologischer Pathologie im Zusammenwirken von Anlage und lußerer Sehlidlichkeit, wobei das Gewicht der Umweltfaktoren vom Autor zweifellos wesentlich höher eingeschátzt wird als die Rolle der mganetischen Matrix ${ }^{H}$; man erinnere sich im abrigen daran, đaB die Umweltfaktoren das mânnliche Prinzip bei der Krankheitszeugung re- 
prasentieren, ein Vergleich, der aus der voremanzipatorischen Epoche des Biedermeier heraus verstanden werden solite. Es handelt sich bei den _causae occasionales" im wesentlichen um eine an die Verhaltnisse der frahindustriellen Gesellschaft adaptierte Fissung der klassischen _sex res nonnaturales", jener Regulativfaktoren der Diatetik, welche bei falschem Gebrauch zu Risiken werden konnen". Die starke Betonung der außjeren Krankbeitsursachen innerhalb einer ontologisehen Pathologie muß als folgerichtig angesehen werden, da hier die Krankheit vom befallenen Organismus (zumindest relativ) unabhangig entsteht. Damit kame STARKS Krankheitstheorie der Entwicklung einer vor allem okologisch orientierten Pathologie entgegen; dieser Traditionsstrang der Krankheitslehre wird jedoch von der anatomisch-morphologischen Pathologie nach 1850 zunachst kaum weitergefohr mit der bedeutsamen Ausnahme RUDOLF VIRCHOWs.

\subsubsection{Zusammenfassung: Pathogenie und Ātiologie im Wandel}

Die ontologische Pathologie und das Analogiemodell eines KArL. WILamm STARK gehoren der Geschichte an; der Versuch einer Pathologie als Physiologie der Krankheit konnte sich nicht behaupten. Statidessen wurde die Entwicklung einer "Pathologischen Physiologie" das Ziel der naturwissenschaftlich orientierten Forscher in der zweiten Halfte des 19. Jahrhunderts - nunmehr unter Leitsatzen wie "Pathologie und Physiologie sind identisch; die Methode beider ist also dieselbe" ${ }^{-52}$ oder „Das Wesen der Krankheit ... ist: AeuBerung der typischen Kraft unter ungewbhnlichen Bedingungen ${ }^{43}$, Pathologische Anatomie, Pathophysiologie, Pathochemie und Experimentelle Pathologie nahmen in den vergangenen 140 Jahren einen ungeahnten Aufschwung, der zu einer Vervielfachung der Detailkenntnisse sowie zu einer Differenzierung und Spezialisierung des Fachgebietes und seiner Fonchungsansaltze fahrte. Grundlagen dieser Entwicklung bildeten die Naturwissenschaften Physik, Chemie, Biologie, Physiologie und Biochemie, die neue Teildisziplinen wie Histopathologie und Zytopathologie erst moglich werden ließen. Im Zentrum der Pathologie stand dabei immer die Sicherung eines morphologisch faßbaren Befundes, des pathologisch-anatomischen Substrats. Vor allem der Krankheitsverlauf, der Wandel von Strukturen und Funktionen, konnte so als formale strukturelle und formale funktionelle Pathogenese beschrieben werden: um die Klärung des Korrelationsmuster von Disposition und Exposition und deren UrsachenWirkung-Gefage bemoht sich die kausale Pathogenese. Weit geringere Fortschritte zeigte hingegen die Atiologie; in einem heutigen Lehrbuch der Pathologie ware die bei STark anzutreffende Relation von Átiologie zu Pathogenese (19:1) undenkbar und eher umzukehren. Ein Vergleich der Begrifle und Inhalte erscheint daher reizvoll und soll hier abschließsend exemplarisch angedeutet werden.

Ein im Jahre 1975 von Rorrek herausgegebenes Lehrbuch der Pathologie fur Medizinstudenten, das auf dem offiziellen Gegenstandskatalog für den Ersten Abschnitt der Arztlichen Prufung basiert, widmet der Allgemeinen Ätiolo- 
gie und Pathogenese 25 von insgesamt 732 Seiten $(3,4 \%$ des Umfangs). Die "Krankheitsanlagen' (causae internae) STARKs haben sich hier zum Begriff der .Disposition' gewandelt, welche als „Krankheitsbereitschaft aufgrund einer... Verminderune der Anpassungsfahigkeit (Adaptation) des Organismus an Stōrungen der Homoostase ${ }^{*}$ definiert wird ${ }^{34}$. Diese Dispositionen lassen sich wiederum untergliedern in

Physiologische Dispositionen

- genetisch fixierte Faktoren

- Geschlechtsdisposition

- Alrersdisposition

Pathologische Dispositionen

- Herabsetzung der unspezifischen Resistenz

- Herabsetzung der spezifischen Resistenz (Immunităt).

Die genetisch fixierten Faktoren (entstanden durch Mutation oder Chromosomenaberration) werden als „endogene Krankheitsursachen" im Abschnitt nAtiologie ${ }^{4}$ noch einmal besonders hervorgehoben ${ }^{35}$. Ein Vergleich mit STARKS „Krankheitsaniagen" zeigt teilweise erstaunliche Obereinstimmungen, doch muß schon hier konstatient werden, dab dessen , causae internae $^{4}$ umfassender konzipiert waren als Rotrens "Dispositionen"

Noch deutlicher wird dieser Befund beim Vergleich der für STARK so eminent wichtigen „Gelegenheitsursachen" (causae occasionales) mit den „exogenen Krankheitsursachen" des modernen Lehrbuches ${ }^{16}$. Was bei STARK 460 Seiten in Anspruch nahm, muB sich 130 Jahre später mit 13 Zeilen begnügen. Als exogene Krankheitsursachen gelten nummehr:

1 belebte Krankheitserreger (Viren, Bakterien, Pilze, Protozoen und vielzellige Organismen) und ihre Toxine

2 quantitativ und/oder qualitativ gestôrte Nahrungszufuhr

3 unzureichende oder abermaßige Sauerstofizufuhr

4 Verletzung (Trauma)

5 Verănderung der Temperatur

6 chemisch-toxische Substanzen

7 Strahlen

8 Veranderung des Luftdrucks

9 Elektrizităt

10 Schilden psychosozialer Art

Tatsächlich werden aber von den genannten Noxen nur die Punkre 1, 3, 5, 6 und 7 im Lehrbuch mit Beispielen abgehandelt, wahrend die pathogenetisch wesentlich problematischeren Bereiche (Nahrung, Luftdruck, Elektrizitalt und psychosoziale Schliden) nur plakativ in der systematischen Obersicht auftau. chen. Diese Eingrenzung det Átiologie auf mit naturwissenschaftichen Methoden quantitativ analysierbare Phänomene kann als gebotene Beschrainkung der exakten Pathologie, aber ebenso als für eine umfassende Krankheitslehre abträglicher ReduktionsprozeB interpretiert werden. Die Aufgabe einer Theoretischen Pathologie besteht unter anderem darin, auf solche historisch erklărba* ren Veränderungen hinzuweisen; dabei kann es allerdings nicht - im Sinne ei- 
nes falsch verstandenen Leitspruchs „Historia magistra vifae" - darum gehen, aberholte Problemlosungsstrategien zu rehabilitieren. Der Wert einer Rackbesinnung auf frahere Konzepte liegt vielmehr im Anfzeigen alter (und damit vielleicht auch wieder kanftiger) Fragestellungen; die Suche nach zeitgemäBen Antworten muB dem in der Forschung tatigen Pathologen überlassen bleiben.

\section{Anmerkungen}

(1) vgl. hierza Foscault, Michel: Die Gebunt der Klinik. Fine Archäologè des ärztichen Blicks. Münches 1973.

(2) siche auch Engellhandt, Dietrich won: Der metaphysiscbe Krankheitsbegriff des Deutuchen Idealivarus. Schellings und Hegrls natarphillowephische Grundieguare in: Seidlet, Edaard $(\mathrm{Hg})$ : Mediainische Anthpopologe. Beitrige far cime Theoretische Pa. thologie. Berlin Heidelberg New Yark Tokyo 1984. S. 17-31.

(3) Bleker, Johanna: Die Naturhiuterische Schule IB25-1545. Ein Reitras zur Geschichte der kinischen Medizin in Deutachland. Stungant 1941. S. 135. Unsere Darstellung der Naturhistorischen Schule lefint sich as diese Monographie an.

(4) siche die Darstellune bei Bleket. Johanna: Die historische Parhologie, Notologie und Epidemiologie im 19. Jahrhundert. In: Medirimbistorisches Journal $79(1984) 33-52$.

(5) Eine gute Dantellune der Epoche ejba Bernhard, Marianne: Das Biedermeier. Ksinur zwischen Wiener Kongrel und Marzrcolution. (Hermes Handlexikan). Disseldorf $198,3$.

(6) Rothschuh, Karl Eiduard: Desische Hiedermeiermedizin. Epoche rwischen Romantik und Naturalismua (1830-1850). in. Gesneris 25 (1968) 167-187, hier 186f.

(ग) Bauer, Axel: Das offentiche Krankenhaus als wissenschaftiche Bildungs-Anstah. Kart Witheim Stark (1787-1845) und sein Konzept eines Akadomischen Lehrkrankenhauses aus dem Jahre 1859 - Ansprech und Wirklichkeit. In: Baunteine zur Medizingeschichie. Heinnich Schipperges zum 65. Gebartuag. Hrag von Eduard Seidler und Heinz Schott. Suthofts Archiv, Deihef 24. Scuttuart 1984. S. 9-17,

(8) w.a. Haeser (18812. Pajel (1898/1915), Neuburger (1903). Diepgen (1951), auch noch Rothechuh (1978).

(9) vor allen Bliker (19s1). Ein Aufratz des Verf, mu diesem Thema befiedet sich im Druck.

(10) vgl. Virchow, Rudotf: Gedachtnisurede auf Johann Lukas Schoniein, gechalten am 23. Ja. nuar 1MES, dem ervien Jahrestage seines Todes, is der Aula der Berliner Universitat. Ber. lin 1M6s. In diener Rede windigt Virchow die Rolle Starks bei der Prägung des Beprifls Krankheitupenenel" (S. 67)

(i) Stark, Kart Wilhelen: Allgriseine Ratbologie ader allgemeine Naturlehre der Krankheit. Zweite, sehr vermehrte und getenserve Aungabe. Dd. I. Leipeie 1844. Bd. 2. Leiprie 1845. Hier zitien Bd. I. S V

(12) sauk (1544) S. IXr

(13) ithid. 8,42

(14) isid. 5. 51

(15) ithid. 5 . 55

(16) ithid. S. S6

(17) Stark, Karl Wilheim: Ueber Indiwidualiar des Krankheitspraerises. In: J. F. C. Heckers

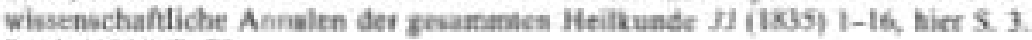

(15) Stark (1844) S. 72

(19) ihid. 5. 73

(20) loc cit

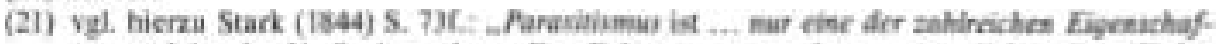
ien. welche der Vorf. als nothwendipe Folgerung aes scinem secicuelichen Begrif der Krankheit fit disse in Ansprach simimt. Man that ihm daher sehr Unrecht, wenn man 
dheses einseine det Krankheit beigelegte Alinibut, wie es geichah, zun Angelpunct und der Basis seiner ganzth Pathologe falschlich machr". (Hervorhebungen von A. B.)

(22) as letaterein vaL. Slark (1835) S. 15F.

(23) Stark (1844) S. 74

(24) ithid S. 105

(25) inid. S. 109 f

(26) ibiat \$. 113

(27) ibid S. 160

(2x) ibiat S. Itos

(29) ibia.s. $205 t$

(D0) Aifhchlulereich fur Starks Einstellung zu seinem eigenen Beruf, vielieicht auch zur Zerstanung gewisser idyllisierender Topoi geeignet, ist die Pasaage íber Arzte (ibid. S. 669). Fi darf angenommen werden, dab dienc pessimistischen Zenlen, nur ein Jahr vor Statks Tod gaschrieben, autnbiographische Zige tragen.

(01) Weilere pathogene Faktoren der atien Heilkunde waren die, ses naturales', res praeter nutuirales' und tra suprananurales', so noch bei Georgius Francus (Gearz Franck van Franckenau) in veinef .Institstionum medicarum 5ynopsis" (Heideibergae 1672), S. $2 \mathrm{kl}$.

(32) Dusucr Satr findet soch bei Henle, lakob. Handouch der rationellen Pathologie. Bd. I. Braunschweic 184t, S. 30 .

(33) ibid 5.97

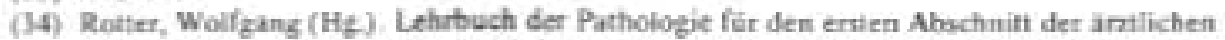
Prufung. 2 Bde. Stutheart/New York 1975. Im folgenden zitiert mach Hd. I, S. 15-1I.

(35) ithod 5 . af

(36) ihid 5.5 